\title{
Periosteal microcirculatory reactions in a zoledronate-induced osteonecrosis model of the jaw in rats
}

\author{
Ágnes Janovszky • Andrea Szabó • Renáta Varga • Dénes Garab • Mihály Boros • \\ Csilla Mester • Nikolett Beretka • Tamás Zombori • Hans-Peter Wiesmann • \\ Ricardo Bernhardt • Imre Ocsovszki • Péter Balázs • József Piffkó
}

Received: 6 August 2014 / Accepted: 22 October 2014 / Published online: 30 October 2014

(C) Springer-Verlag Berlin Heidelberg 2014

\begin{abstract}
Objectives Nitrogen-containing bisphosphonates induce osteonecrosis mostly in the jaw and less frequently in other bones. Because of the crucial role of periosteal perfusion in bone repair, we investigated zoledronate-induced microcirculatory reactions in the mandibular periosteum in comparison with those in the tibia in a clinically relevant model of bisphosphonate-induced medication-related osteonecrosis of the jaw (MRONJ).

Materials and methods Sprague-Dawley rats were treated with zoledronate (ZOL; 80 i.v. $\mu \mathrm{g} / \mathrm{kg} /$ week over 8 weeks) or saline vehicle. The first two right mandibular molar teeth were extracted after 3 weeks. Various systemic and local (periosteal) microcirculatory inflammatory parameters were examined by intravital videomicroscopy after 9 weeks.
\end{abstract}

Ágnes Janovszky and Andrea Szabó contributed equally to this work.

Á. Janovszky $\cdot$ R. Varga $\cdot$ J. Piffkó

Department of Oral and Maxillofacial Surgery, University of Szeged,

Szeged, Hungary

\author{
A. Szabó $(\bowtie) \cdot$ D. Garab • M. Boros • C. Mester • N. Beretka \\ Institute of Surgical Research, University of Szeged, \\ Szőkefalvi-Nagy Béla 6, Szeged H-6720, Hungary \\ e-mail: szabo.andrea.exp@med.u-szeged.hu \\ T. Zombori \\ Department of Pathology, University of Szeged, Szeged, Hungary \\ H.-P. Wiesmann • R. Bernhardt \\ Institute of Materials Science, Max Bergmann Center of \\ Biomaterials, TU Dresden, Dresden, Germany \\ I. Ocsovszki \\ Department of Biochemistry, University of Szeged, Szeged, Hungary \\ P. Balázs \\ Department of Image Processing and Computer Graphics, University \\ of Szeged, Szeged, Hungary
}

Results Gingival healing disorders $(\sim 100 \%)$ and MRONJ developed in $70 \%$ of ZOL-treated cases but not after saline (shown by micro-CT). ZOL induced significantly higher degrees of periosteal leukocyte rolling and adhesion in the mandibular postcapillary venules (at both extraction and intact sites) than at the tibia. Leukocyte NADPH-oxidase activity was reduced; leukocyte CD11b and plasma TNF-alpha levels were unchanged.

Conclusion Chronic ZOL treatment causes a distinct microcirculatory inflammatory reaction in the mandibular periosteum but not in the tibia. The local reaction in the absence of augmented systemic leukocyte inflammatory activity suggests that topically different, endothelium-specific changes may play a critical role in the pathogenesis of MRONJ.

Clinical relevance This model permits for the first time to explore the microvascular processes in the mandibular periosteum after chronic ZOL treatment. This approach may contribute to a better understanding of the pathomechanism and the development of strategies to counteract bisphosphonate-induced side effects.

Keywords Mandibular periosteum · Intravital fluorescence videomicroscopy $\cdot$ Leukocytes · Inflammation .

Bisphosphonate $\cdot$ Osteonecrosis

\section{Introduction}

Bisphosphonates (BISs) are widely used for the treatment of osteoporosis and tumors with bone metastasis. The therapeutic effect is linked to the inhibition of osteoclast activity, which alters the bone metabolism by inhibiting bone resorption and reducing the bone turnover [1]. Although BIS treatment undoubtedly improves the quality of life of the patients, osteonecrosis is a serious adverse effect in a number of cases [2]. BIS-related osteonecrosis of the jaw (recently termed as 
medication-related osteonecrosis of the jaw; MRONJ) occurs mainly after invasive dental procedures, e.g., tooth extraction [3], with an increased incidence particularly after the use of third-generation BISs (e.g., zoledronate, ZOL) [1]. MRONJ most probably has a multifactorial etiology and is influenced by numerous factors, including the administration route and dose, the duration of the therapy, the indication of BIS administration (osteoporosis or oncological reason), co-morbidities, the concomitant use of other drugs (corticosteroids or chemotherapeutics), genetic factors, age, and poor oral hygiene [1, 3]. Local contamination and infection evoked by invasive dental procedures in the presence of BIS treatment have also been emphasized in the development of MRONJ [4]. Osteonecrosis, however, can develop several years later, which may be explained by the long half-lives of these medications [1] and not by the acute infectious induction. Moreover, BIS treatment has been shown to cause sterile inflammatory reactions such as aseptic peritonitis $[5,6]$ and an enhancement of leukocyte-endothelial cell interactions in the knee joint [7]. These effects may be linked to an upregulation of pro-inflammatory cytokines such as IL-1 and TNFalpha [6-8] in response to BIS administration. The effects of BISs also exhibit spatial differences, because certain inflammatory reactions are confined to the mandible and not present in the femur [9]. Nevertheless, the exact pathomechanism of MRONJ has not yet been clarified, and the possibilities of its prevention or the use of curative modalities are also limited.

The periosteal perfusion significantly influences bone healing and determines the prognosis of adjacent soft tissue traumas as well [10]. Little, however, is known about the microcirculatory effects of BIS and especially the microcirculation of the mandible. Likewise, to date, no data are available on the periosteal changes after invasive dental procedures involving BIS treatment. In this study, we hypothesized that a disturbed mandibular microcirculation may play a role in the pathogenesis of MRONJ. With this background, we designed an animal model of MRONJ with the possibility of visualizing the mandibular microcirculation by means of an intravital videomicroscopy (IVM) technique. Our aims were to observe and compare the mandibular and tibial periosteal microcirculatory reactions in rats subjected to chronic ZOL treatment with or without tooth extraction.

\section{Materials and methods}

All chemicals were purchased from Sigma-Aldrich (St. Louis, MO, USA) unless indicated otherwise. The study was performed in accordance with the Guidelines laid down by the National Institute of Health (NIH) in the USA regarding the care and use of animals for experimental procedures and with the 2010/63/EU Directive and was approved by the Animal
Welfare Committee of the University of Szeged (V/1639/ 2013).

\section{Experimental protocol}

Twenty male Sprague-Dawley rats (average initial body weight of $200 \pm 10 \mathrm{~g}$ ) were randomly allocated to saline vehicle-treated control $(n=10)$ or intravenously (i.v.) ZOLtreated $(n=10, \mathrm{ZOL})$ groups. ZOL (zoledronic acid, Zometa, Novartis Europharm, Budapest, Hungary) was administered through the tail vein in a dose of $80 \mu \mathrm{g} / \mathrm{kg}$ once a week for 8 weeks. At the end of the 3 rd week of the protocol, the first and second molar teeth on the right side were extracted from the mandible under ketamine and xylazine (i.p. 25 and $75 \mathrm{mg} / \mathrm{kg}$, respectively) anesthesia. The teeth were luxated with an $18 \mathrm{G}$ needle, and the extraction was performed with extraction forceps. The roots were also removed with a dental drill under a Zeiss operating microscope $(\times 6$ magnification; Carl Zeiss GmbH, Jena, Germany). By these means, the defect was equal in size and severity in all rats. For pain relief, intramuscular ketoprofen (Ketodex Forte; Berlin-Chemie AG, Berlin, Germany; $5 \mathrm{mg} / \mathrm{kg}$ ) and oral metamizole sodium (Algopyrin; Sanofi-Aventis, Budapest, Hungary; $75 \mathrm{mg} / \mathrm{kg}$ ) were administered for 3 days. Mucosal healing processes were monitored continuously throughout the experimental period.

Microcirculatory variables were examined on the 9th week of the protocol. The animals were anesthetized intraperitoneally with an initial dose of sodium pentobarbital $(45 \mathrm{mg} / \mathrm{kg})$ and placed in a supine position on a heating pad to maintain the body temperature at $36-37^{\circ} \mathrm{C}$. Following cannulation of the trachea, the penile vein was cannulated for the administration of fluid and drugs (supplementary dose of sodium pentobarbital; $5 \mathrm{mg} / \mathrm{kg}$ ). This was followed by cannulation of the femoral artery on the right side, and blood was drawn for the white blood cell count and determination of the different markers of leukocyte function/activation and inflammation (see later).

The mandibular periosteum was exposed for fluorescence IVM on both sides, in the vicinity of the earlier extraction area and on the contralateral side, between the anterior part of the deep masseter and the anterior superficial masseter muscles, as described elsewhere [11]. Briefly, an incision was made parallel to the incisor tooth in the facial skin and the underlying subcutaneous tissue, and the loose connective tissue between the fascia of the deep masseter and the anterior superficial masseter muscles was carefully cut, using a microsurgical approach under an operating microscope $(\times 6$ magnification; Carl Zeiss GmbH, Jena, Germany). By this means, the periosteal membrane covering the corpus of the mandible at the anterior margin of the molar region was reached, laterally/ distally to the incisor tooth. To aid better exposure for the microscope objective, retraction was achieved by placing 
stitches with 7.0 monofilament polypropylene microsurgical thread into the surrounding masseter muscles. For comparison of the characteristics of the mandibular microcirculation with those of the tibial periosteum, the medial/anterior surface of the left tibia was exposed by complete transection of the anterior gracilis muscle with microscissors and careful atraumatic microsurgical removal of the connective tissue covering the tibial periosteum [12]. After the IVM recordings of the microcirculation, the animals were over-anesthetized with a single overdose of pentobarbital, and the mandibles were removed and placed into $10 \%$ buffered formalin solution for subsequent detection of osteonecrosis of the mandible through micro-CT and histological analyses.

\section{Fluorescence IVM}

The exposed periosteal surfaces of the mandible (on both the extracted and intact sides) and of the tibia were consecutively examined by IVM. The exposed surfaces were positioned horizontally on an adjustable stage and superfused with $37{ }^{\circ} \mathrm{C}$ saline. The periosteal microcirculation was visualized by IVM (penetration depth: approx. $250 \mu \mathrm{m}$; Zeiss Axiotech Vario 100HD microscope; 100-W HBO mercury lamp; Acroplan 20×/ 0.5 N.A. W, Carl Zeiss GmbH, Jena, Germany). Fluorescein isothiocyanate-labeled erythrocytes $(0.2 \mathrm{ml}$ i.v.) were used to stain red blood cells and rhodamine-6G $(0.2 \%, 0.1 \mathrm{ml}$ i.v. $)$ to stain leukocytes. Images from four to five fields of the mandibular and the tibial periosteum from each rat were recorded with a chargecoupled device video camera (Teli CS8320Bi, Toshiba Teli Corporation, Osaka, Japan) attached to an S-VHS video recorder (Panasonic AG-MD 830; Matsushita Electric Industrial Co., Tokyo, Japan) and a personal computer.

\section{Video analysis}

Quantitative evaluation of the microcirculatory parameters was performed off-line by the frame-to-frame analysis of the videotaped images taken for IVM (IVM Software; Pictron Ltd, Budapest, Hungary). Leukocyte-endothelial cell interactions were analyzed in at least four postcapillary venules per rat. Rolling leukocytes were defined as cells moving with a velocity less than $40 \%$ of that of the erythrocytes in the centerline of the microvessel and passing through the observed vessel segment within $30 \mathrm{~s}$ and are given as the number of cells per second per vessel circumference. Adherent leukocytes were defined as cells that did not move or detach from the endothelial lining within an observation period of $30 \mathrm{~s}$ and are given as the number of cells per square millimeter of endothelial surface, calculated from the diameter and length of the vessel segment. Red blood cell velocity $(\mathrm{RBCV}, \mu \mathrm{m} / \mathrm{s})$ was determined by frame-to-frame analysis of five to six consecutive video-captured images taken after labeling of the erythrocytes.

\section{NADPH-oxidase activity of neutrophil leukocytes}

The NADPH-oxidase activity of the isolated leukocytes was determined by a modified chemiluminometric method described by Bencsik et al. [13]. Blood was drawn from the femoral artery into EDTA-containing tubes, and the erythrocytes in $100 \mu \mathrm{l}$ of whole blood were lyzed in a hypotonic solution and centrifuged at $2000 \mathrm{~g}$. The pellet was resuspended and washed twice in a Dulbecco's phosphate-buffered saline solution. Twenty microliters of resuspended pellet was incubated for $3 \mathrm{~min}$ at $37{ }^{\circ} \mathrm{C}$ in Dulbecco's solution containing lucigenin (1 mM), EGTA (1 $\mathrm{mM})$ and saccharose $(140 \mathrm{mM})$. NADPH-oxidase activity was determined via the NADPH-dependent increase in luminescence elicited by adding $100 \mathrm{mM}$ NADPH (in $20 \mu \mathrm{l}$ ), measured with an FB12 Single Tube Luminometer (Berthold Detection Systems GmbH, Bad Wildbad, Germany). Samples incubated in the presence of nitroblue tetrazolium served as controls. The measurements were performed in triplicates and were normalized for protein content.

\section{Whole blood free radical production}

Ten microliters of blood dissolved in Hanks buffer was incubated for $20 \mathrm{~min}$ at $37{ }^{\circ} \mathrm{C}$ in lucigenin $(5 \mathrm{mM}$; dissolved in Hanks buffer) or luminol (15 mM; dissolved in Hanks buffer) solution in the presence or absence of zymozan $(190 \mu \mathrm{M}$, dissolved in Hanks buffer). Superoxide and hydrogen peroxide production were estimated via the zymozan-induced increase in chemiluminescence (measured with the above luminometer) and normalized for leukocyte counts in the peripheral blood.

\section{Expression of CD11b adhesion molecule on neutrophil leukocytes}

The surface expression of CD11b on the peripheral blood granulocytes was determined by flow cytometric analysis as detailed elsewhere [12], with a CyFlow ML (Partec GmbH, Münster, Germany). 


\section{Plasma TNF-alpha content}

Blood samples were centrifuged at $13.500 \mathrm{rpm}$ for $5 \mathrm{~min}$ at $4{ }^{\circ} \mathrm{C}$ and then stored at $70{ }^{\circ} \mathrm{C}$ until assayed. Plasma TNF-alpha concentrations were determined in duplicate by means of a commercially available ELISA kit (R\&D Systems, Minneapolis, MN, USA).

\section{Evaluation of the gingival lesions}

Healing of the gingiva at the end of the study period (6 weeks after the tooth extraction) was determined on the basis of an osteonecrosis staging system provided by the American Association of Oral and Maxillofacial Surgeons [3]; this was adapted for rats (see Table 1). The examination was performed under an operating microscope $(\times 6$ magnification; Carl Zeiss $\mathrm{GmbH}$, Jena, Germany) by an independent maxillofacial surgeon. The incidence and the severity of the gingival healing disorder were evaluated simultaneously.

\section{Mandibular osteonecrosis as determined by micro-CT}

Mandibles fixed with formaldehyde were used for micro-CT imaging (SCANCO vivaCT 75; Scanco Medical, Brüttisellen, Switzerland); subsequent analysis was performed on 2D sections in the coronal view of the images, the section being chosen that showed the highest degree of tissue defect at the earlier extraction site. The mean density of the bone was estimated via the calculated percentage of the radiolucent area of the alveolar portion of the bone.

\section{Mandibular osteonecrosis as determined by histology}

The specimens were fixed in $6 \%$ neutral buffered formalin for 10 days, then rinsed in phosphate-buffered saline and decalcified in $5 \%$ EDTA for 7 days. The decalcified specimens were embedded in paraffin and cut into 20 semi-serial sections with a microtome (Shandon Finesse 325; Thermo Scientific, Waltham, MA, USA), and routine hematoxylin

Table 1 Scoring of macroscopic signs of the bisphosphonate-related healing processes after tooth extraction (adopted from the staging of MRONJ by Ruggiero et al. [3])

\begin{tabular}{llll}
\hline Score & Exposed bone & Inflammation/infection & Fistula formation \\
\hline Score 0 & - & - & - \\
Score 1 & + & - & - \\
Score 2 & + & + & - \\
Score 3 & + & + & + \\
\hline
\end{tabular}

and eosin (H\&E) staining was performed. The sections were examined under a light microscope at $\times 4-40$ magnification (Model CHT; Olympus, Hamburg, Germany). The incidence of osteonecrosis of the jaw was determined on the basis of characteristic signs of necrosis, such as missing nuclear staining, the development of sequester formation and inflammatory infiltration.

\section{Statistical analysis}

The statistical analysis was performed with a statistical software package (SigmaStat for Windows; Jandel Scientific, Erkrath, Germany). For the analysis of microcirculatory parameters, changes in variables within and between groups (with respect to location and treatment, separately) were analyzed by the two-way ANOVA test, followed by the Holm-Sidak test. Differences between groups (other inflammatory parameters and scores) were analyzed with the Student $t$ test. Data are presented as mean values and SEM in all Figures and Tables. $P$ values $<0.05$ were considered significant.

\section{Results}

Microcirculatory inflammatory reactions

IVM recordings of the microcirculation were performed in a mandibular periosteal region just adjacent to the site of the

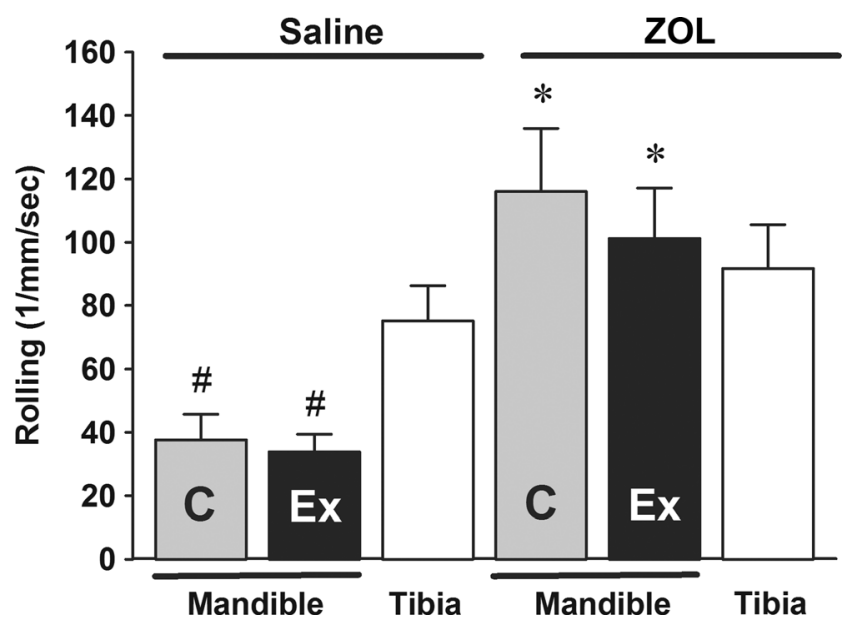

Fig. 1 Periosteal primary leukocyte-endothelial cell interactions (rolling) in saline- and ZOL-treated animals in the postcapillary venules of the mandible on the tooth extraction $(E x)$ and the contralateral $(C)$ sides and in the tibia. Data are presented as means \pm SEM. Asterisk indicates $P<0.01$ vs. the corresponding saline-treated group. The pound sign indicates $P<0.05$ vs. the tibia. Two-way ANOVA was followed by the Holm-Sidak test 


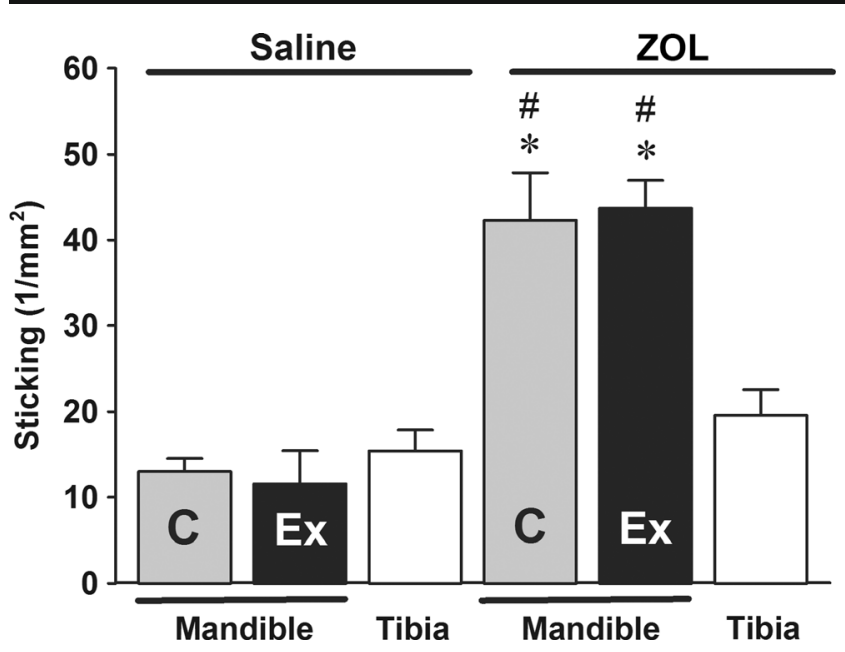

Fig. 2 Periosteal secondary leukocyte-endothelial cell interactions (sticking) in the postcapillary venules of the mandible on the tooth extraction $(E x)$ and the contralateral $(C)$ sides and in the tibia in salineand ZOL-treated animals. Data are presented as means \pm SEM. Asterisk indicates $P<0.01$ vs. the corresponding saline-treated group. The pound sign indicates $P<0.01$ vs. the tibia. Two-way ANOVA was followed by the Holm-Sidak test

earlier tooth extraction and also on the contralateral side. Data were compared with those on the tibial periosteum.

In vivo microscopy revealed homogenous microvascular perfusion in all of the periosteal tissues examined; the RBCVs were similar in the mandibular and tibial capillary beds $(827.5 \pm 30.1 \mu \mathrm{m} / \mathrm{s}$ and $739.0 \pm 37.7 \mu \mathrm{m} / \mathrm{s}$, respectively). The data were similar on the two sides of the mandible and were not influenced by chronic ZOL treatment (data not shown).

However, the leukocyte rolling in the postcapillary venules of the mandible in the ZOL-treated group was significantly higher than in the saline-treated group both at the site of tooth extraction and on the contralateral side; the differences between the sites were not statistically significant (Fig. 1). Similar differences were observed in the leukocyte adhesion values after ZOL, which revealed a statistically significant enhancement in the mandibular periosteum as compared with the tibial periosteum (Fig. 2). ZOL evoked similar rolling and adhesion values irrespectively of the presence of MRONJ (data not shown). The tibial microcirculation was characterized by higher leukocyte rolling but similar adhesion in comparison with the data obtained for the mandible in the saline-treated animals; none of them were influenced by $\mathrm{ZOL}$ at this location.

Free radical production of leukocytes

The NADPH-oxidase activity of the neutrophil leukocytes harvested from ZOL-treated animals was significantly lower than that from the control animals (Fig. 3a). The free radical-derived chemiluminescence of the whole blood (as determined by the superoxide and hydroxyl radical-dependent chemiluminescence measurements) indicated no differences between the two experimental groups (Fig. 3b).

\section{Other inflammatory parameters}

To exclude the possibility of increased leukocyte counts behind the increased PMN rolling and adhesion after ZOL treatment, the number of PMNs was determined with the conventional Türk solution staining method and using a hemocytometer. As expected, the number of PMN leukocytes was not higher (but rather even lower) in the rats chronically treated with ZOL (Table 2).

As evidenced by the mean fluorescence values of the adhesion molecule CD11b within the leukocyte population (as measured by flow cytometry), no significant differences were detected between the saline- and ZOL-treated animals (Table 2).

There were no differences between the saline- and ZOLtreated experimental groups with respect to the plasma TNFalpha levels either ( $n=6$ and $n=5$, respectively) (Table 2).
Fig. 3 The effects of chronic ZOL treatment on leukocyte NADPH-oxidase activity (a) and whole blood free radical production (b) (the latter shown by chemiluminescence in the presence of lucigenin and luminol to detect superoxide anion and hydroxyl radical production, respectively). Data are presented as means \pm SEM. Asterisk indicates $P<0.05$ vs. saline, Student $t$ - test

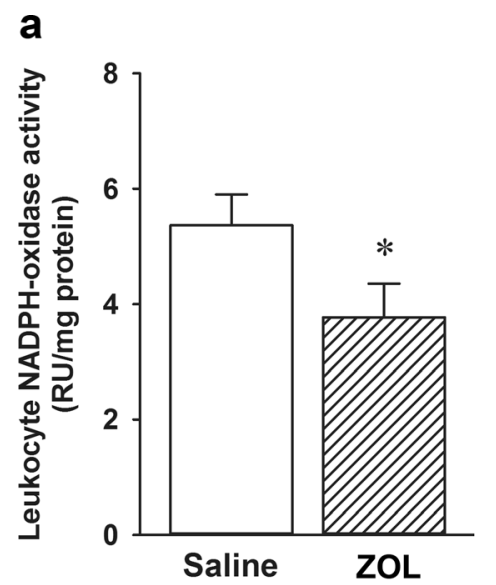

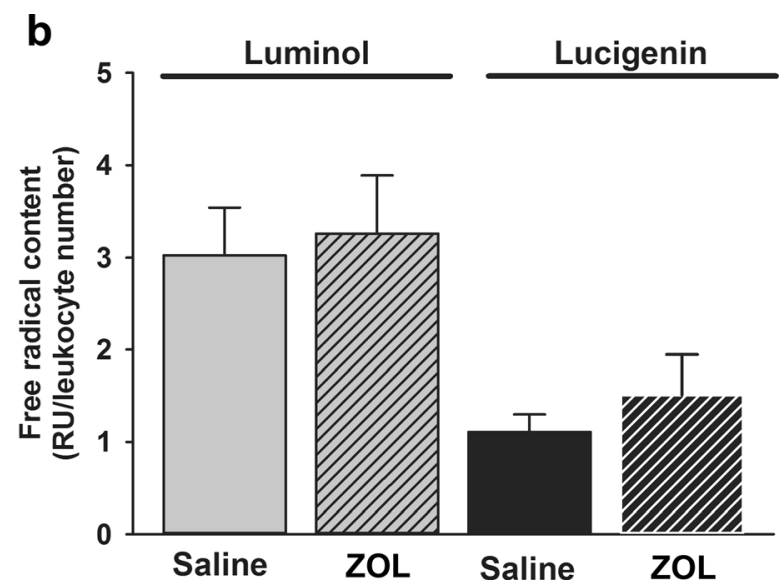


Table 2 The effects of chronic ZOL treatment on the leukocyte count, neutrophil-derived $\mathrm{CD} 11 \mathrm{~b}$ adhesion molecule expression and plasma TNF-alpha levels

\begin{tabular}{llll}
\hline Parameter & Saline & ZOL & $P$ values \\
\hline $\begin{array}{c}\text { PMN leukocyte count in } \\
\text { the blood (cells/ } \mu \mathrm{l})\end{array}$ & $4513 \pm 250$ & $3731 \pm 215$ & $<0.05$ \\
$\begin{array}{c}\text { CD11b expression (mean } \\
\text { fluorescence intensity) }\end{array}$ & $1.57 \pm 0.21$ & $1.37 \pm 0.09$ & n. s. \\
\begin{tabular}{l} 
TNF-alpha (pg/ml) \\
\hline
\end{tabular} & $2.65 \pm 0.49$ & $2.33 \pm 0.39$ & n. s. \\
\hline
\end{tabular}

Data are presented as mean \pm SEM. $P<0.05$ vs. saline, Student $t$ test n. s. not significant

Gingival healing after tooth extraction

Six weeks after the tooth extraction, intact mucosa could be observed in $8 / 10$ of the control animals (the average healing score was $0.25 \pm 0.25$ ), but different degrees of mucosal healing disorders were detected in all (10/10) of the ZOLtreated animals. The severity of the healing disorders reached a score of $1.83 \pm 0.18$ in this group $(p<0.01)$.

\section{Incidence and severity of mandibular osteonecrosis}

Normal bony regeneration with a radiolucent areas of $12.09 \pm$ $1.91 \%$ of the alveolar bone could be detected at the site of the earlier tooth extraction in all (10/10) of the saline-treated animals. In contrast, a certain degree of discontinuity of the cortical and spongious bone regions was found in $7 / 10$ of the ZOL-treated animals (Fig. 4). This higher incidence of impaired bony regeneration was accompanied by a significantly lower average bone density in this group $(39.51 \pm 7.18 \%$ of the alveolar area) as compared with that in the saline-treated group $(p<0.01)$.

The radiological diagnosis of mandibular osteonecrosis was confirmed by standard histological examinations (Fig. 5). Findings of missing nuclear staining in the osteocytes increased inflammatory infiltration and granulation tissue formation around the necrotic area, and occasional sequester formation were made in $6 / 10$ of the ZOL-treated animals, whereas nearly normal bone regeneration was observed in the other rats.

\section{Discussion}

The major aim of the present study was to examine the mandibular periosteal microcirculatory reactions in a rodent model of MRONJ. Through the chronic administration of high i.v. doses of ZOL in combination with an invasive dental intervention, a high prevalence of mucosal healing disorders $(\sim 100 \%)$ was achieved together with a relatively high osteonecrosis rate $(70 \%$; as revealed by micro-CT and histological analyses). This protocol was based on a modified literature method [14]. BIS doses in the range $20-2250 \mu \mathrm{g} / \mathrm{kg}$ with different frequencies and different administration routes have been administered by others (for a meta-analysis [15]). The relatively high dose applied here $(80 \mu \mathrm{g} / \mathrm{kg} /$ week $)$ is still well tolerated in rats, and although it was also administered in a higher frequency than on human use, it produced symptoms and radiological evidence similar to those observed in humans. Apart from the dose of ZOL, the relatively high incidence of MRONJ in this study can be explained by (1) the triggering effect of the applied dental extraction (the a

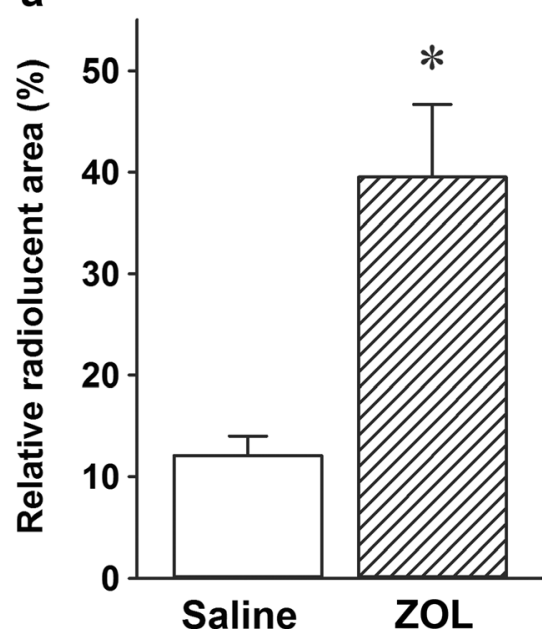

Fig. 4 Bone density differences expressed as a percentage of the radiolucent area of the alveolar bone (marked with a rectangle) in saline- and BIS-treated animals 6 weeks after tooth extraction (a). Data are presented

\section{b}

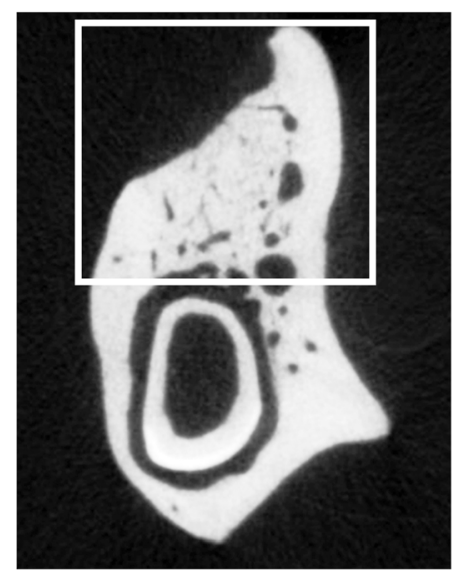

C

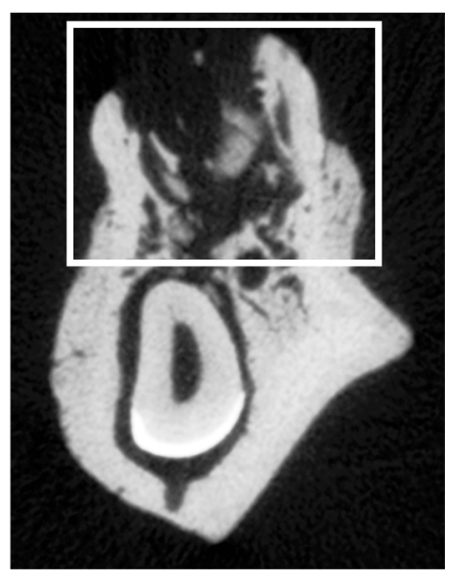

as means \pm SEM. Asterisk indicates $P<0.05$ vs. saline, Student $t$ test. Micro-CT scans show representative images of the mandibular crosssections in saline- and ZOL-treated rats (b and c, respectively) 


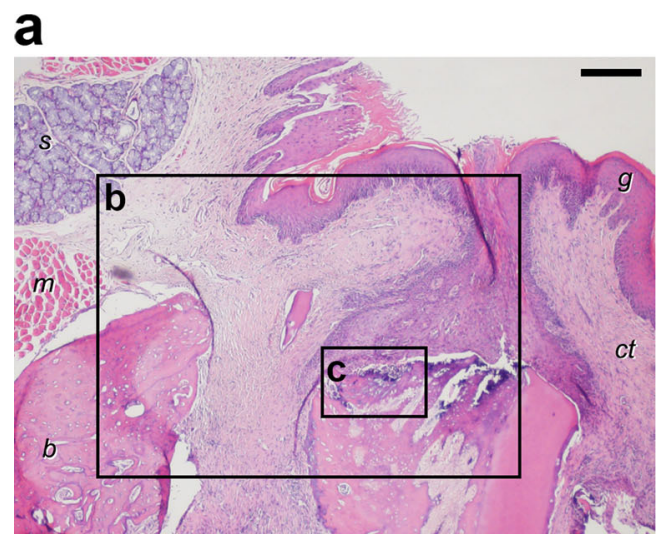

b
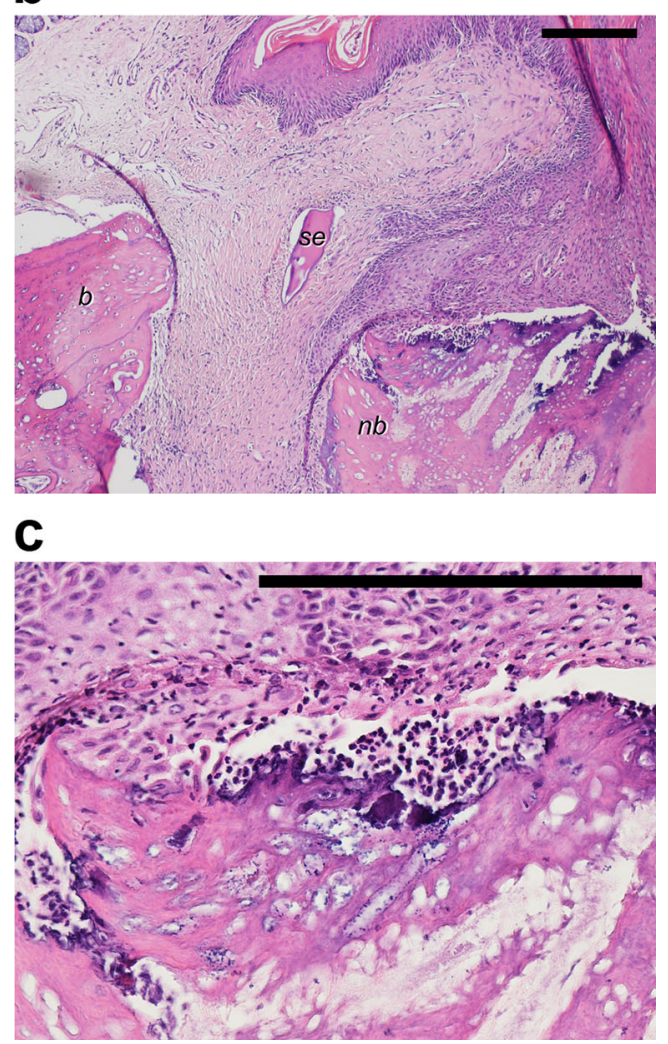

Fig. 5 Representative micrograph (H\&E staining) showing regeneration processes in a ZOL-treated animal 6 weeks after tooth extraction (magnification $\times 4)($ a). $s$ salivary gland, $m$ muscle, $b$ bone, $g$ gingiva, $c t$ connective tissue. Sequester formation ( $s e$ ) and lack of nuclear staining of the necrotic bone $(n b)$ and PMN granulocyte infiltration around the necrotic area (center of the section) can be seen at higher magnifications (magnifications $\times 10$ and 40 ) (b, and c, respectively). The bar denotes $200 \mu \mathrm{m}$

importance of which has been demonstrated in MRONJ patients) [3]) and (2) the use of the mandibular site (there is a higher prevalence of osteonecrosis at this localization in humans) [16].

It is reasonable to assume that impaired regeneration processes contribute to the pathophysiology of MRONJ. From a functional aspect, bony regeneration processes depend not only on the functional activity of the osteoblasts and osteoclasts but also on the blood supply and angiogenesis. BISs have been shown to influence all of these processes. As such, the inhibition of osteoclast recruitment to the bone surface [17] and shortening of the osteoclast life span are the main effects of BISs that are brought about directly or indirectly (via the OPG-RANKL pathway) [18]. Accordingly, delayed bone healing $[19,20]$, together with decreased bone formation and vascularity in the extraction socket, have been detected in ZOL-treated rats [21]. Numerous studies have elucidated the antiangiogenic effects of BIS both in vitro [22] and in vivo [20,23]. Furthermore, thicker and less connected/ordered blood vessels in the alveolar bone of the mandible were found in ZOL-treated rats after tooth extraction [24]. The aim of the present study was to assess not the structural but the functional aspects of chronic BIS treatment on the microvasculature.

Direct toxic and inflammatory effects of BISs may also contribute to the development of MRONJ. BISs exert toxic effects on many different cell types (fibroblasts, osteoblasts, and endothelial and epithelial cells), manifested in diminished cell proliferation and decreased collagen production, ZOL being the most inhibitory in this respect [25-27]. Furthermore, marked inflammatory reactions are attributed to BISs through the induction of peritonitis via the activation of immunological pathways after intraperitoneal administration [5, 6, 28]. Enhanced leukocyte-endothelial interactions have been demonstrated by means of IVM after BIS treatment in an arthritis model in mice [7]. BIS-associated inflammatory bony changes have also been detected in the mandible [9, 29]. Interestingly, these inflammatory changes were limited to the mandible and were not seen in the femur or the tibia $[9,29]$. Highdose ZOL exacerbates the inflammatory response in a periodontitis model, where the bone lesions strikingly resemble MRONJ [21]. In the present study, pro-inflammatory aspects of chronic BIS treatment could also be traced in the mandibular periosteum, and histological analysis supported the infiltration of the tissue by leukocytes in the neighboring necrotic zone.

In this microsurgical model, the periosteal microcirculation of the mandible can be visualized relatively easily in the molar region, which is likewise a cardinal localization of MRONJ [3]. Apart from nutritive considerations, the periosteum is important for its osteoprogenitor cell content during bone regeneration. Although BISs exert effects on osteoblast proliferation, differentiation and migration in the entire skeleton [30], their action seems to depend on the anatomical location, with the jawbones as highly frequent sites of osteonecrosis. After prolonged use, BISs are known to accumulate in the skeleton, reaching the highest concentration in the mandible $[25,31]$, which may explain their potential toxic effects predominantly in the jawbones. Furthermore, osteoblasts have different proliferation properties at different locations (appendicular vs. axial bones) under physiological circumstances, 
and this phenomenon is also critically influenced by BIS treatment [32]. The functional activity of the osteocytes too differs between the mandible and the tibia [33], and the aggravating effects of BISs on bone healing are confined to the jaw [34]. Although the above findings reveal certain potential factors contributing to the higher incidence of osteonecrosis of the jawbones, the exact pathomechanism is unknown.

As opposed to the microcirculatory consequences of bone injury (i.e. fractures) [35], the effects of tooth extraction on the microcirculatory derangement and local inflammation are less commonly described, due to methodological constraints. We focus here on the microcirculatory aspects of chronic ZOL treatment combined with an earlier local trauma of the jaw (tooth extraction). IVM data were obtained in the proximity of the injury and from a contralateral (intact) site on the mandibular periosteum and were compared with those relating to the intact tibia. After chronic ZOL treatment, increased degrees of leukocyte-endothelial interactions (rolling and adhesion) were observed in the mandibular periosteum, both at the site of the earlier tooth extraction and at the contralateral site, but the corresponding interactions in the tibia were less extensive. It is still an unanswered question why the examined cell-tocell interactions are higher in the postcapillary venules of the mandible, irrespectively of the proximity of the tooth extraction site and the presence of MRONJ in the ZOL-treated group. In preliminary studies, we did not observe inflammatory complications in the mandibular periosteum without tooth extraction, which demonstrated the triggering effect of the trauma in this region. This observation was supported by further findings, when more intense inflammatory reactions of ZOL were evolved in the acute phase after tooth extraction (data not shown). The inflammatory processes were similarly shown in an IVM study to be aggravated by a BIS in an arthritis model in mice [7]. Elevated levels of the proinflammatory cytokine TNF-alpha have been reported in human patients in response to certain types of BISs [8] but were not detected after the chronic administration of a BIS in our study. Furthermore, the number and functional activity (free radical-producing capacity) of PMNs were moderately reduced here. Such effects on the free radical-producing potential of PMNs (including NADPH-oxidase activity) have also been demonstrated by others [36, 37]. Favot et al. suggested that the compromised neutrophil functions, too, may be used as potential biomarkers for MRONJ susceptibility [38]. Interestingly, others have found impaired neutrophil chemotaxis after BIS exposure in mice [36] and humans [38], and this parameter is influenced most extensively by ZOL among the different types of BISs [39]. For leukocyte-endothelial interactions (as seen in our study), an enhanced expression of adhesion molecules is required on the surface of the endothelial cells and/or neutrophil leukocytes [40]. Interestingly, expression of the neutrophil-derived adhesion molecule CD11b (responsible for leukocyte adherence) was not found to be influenced by chronic ZOL treatment here or in other studies. The extents of these inflammatory reactions, however, differed in the jaw and the tibial regions. These regional differences might be explained by different degrees of endotheliumderived adhesion molecule expression at the different anatomical locations.

\section{Conclusions}

A causative relationship between the microcirculatory inflammatory reactions and the pathogenesis of MRONJ could not be provided in the present study; regional differences in endothelial function/dysfunction, however, may contribute to the explanation of differences in the occurrence of osteonecrosis seen at different anatomical locations.

Acknowledgments This publication is supported by the European Union and co-funded by the European Social Fund. Research grants: TÁMOP 4.2.4. A/2-11-1-2012-0001 "National Excellence ProgramElaborating and operating an inland student and researcher personal support system convergence program;" TÁMOP 4.2.1/B-09/1/KONV2010-0005; TÁMOP 4.2.2A-11/1/KONV-2012-0035; TÁMOP 4.2.2A11/1/KONV-2012-0073 "Telemedicine-focused research activities on the field of Mathematics, Informatics and Medical sciences," and OTKA 109388 .

Conflict of interest The authors declare that they have no conflict of interest.

\section{References}

1. Brozoski MA, Traina AA, Deboni MC, Marques MM, NaclérioHomem Mda G (2012) Bisphosphonate-related osteonecrosis of the jaw. Rev Bras Reumatol 52(2):265-270. doi:10.1590/S048250042012000200010

2. Marx RE (2003) Pamidronate (Aredia) and zoledronate (Zometa) induced avascular necrosis of the jaws: a growing epidemic. J Oral Maxillofac Surg 61(9):1115-1117. doi:10.1016/S0278-2391(03) 00720-1

3. Ruggiero SL, Dodson TB, Assael LA, Landesberg R, Marx RE, Mehrotra B (2009) Task force on bisphosphonate-related osteonecrosis of the jaws, American Association of Oral and Maxillofacial Surgeons (2009) American Association of Oral and Maxillofacial Surgeons position paper on bisphosphonate-related osteonecrosis of the jaw-2009 update. Aust Endod J 35(3):119 130. doi:10.1111/j.1747-4477.2009.00213.x

4. Wei X, Pushalkar S, Estilo C, Wong C, Farooki A, Fornier M, Bohle G, Huryn J, Li Y, Doty S, Saxena D (2012) Molecular profiling of oral microbiota in jawbone samples of bisphosphonate-related osteonecrosis of the jaw. Oral Dis 18(6):602-612. doi:10.1111/j. 1601-0825.2012.01916.x

5. Calligeros D, Douglas P, Abeygunasekera S, Smith G (1993) Aseptic peritonitis in association with the use of pamidronate. Med J Aust 159(2): 144

6. Norton JT, Hayashi T, Crain B, Corr M, Carson DA (2011) Role of IL-1 receptor-associated kinase-M (IRAK-M) in priming of immune 
and inflammatory responses by nitrogen bisphosphonates. Proc Natl Acad Sci U S A 108(27):11163-11168. doi:10.1073/pnas. 1107899108

7. Zysk SP, Dürr HR, Gebhard HH, Schmitt-Sody M, Refior HJ, Messmer K, Veihelmann A (2003) Effects of ibandronate on inflammation in mouse antigen-induced arthritis. Inflamm Res 52(5):221226. doi: $10.1007 / \mathrm{s} 000110300075$

8. Anastasilakis AD, Polyzos SA, Makras P, Sakellariou GT, Bisbinas I, Gkiomisi A, Delaroudis S, Gerou S, Ballaouri I, Oikonomou D, Papapoulos SE (2012) Acute phase response following intravenous zoledronate in postmenopausal women with low bone mass. Bone 50(5):1130-1134. doi:10.1016/j.bone.2012.02.006

9. Senel FC, Kadioglu Duman M, Muci E, Cankaya M, Pampu AA, Ersoz S, Gunhan O (2010) Jaw bone changes in rats after treatment with zoledronate and pamidronate. Oral Surg Oral Med Oral Pathol Oral Radiol Endod 109(3):385-391. doi:10.1016/j.tripleo.2009.10. 011

10. Schaser KD, Zhang L, Haas NP, Mittlmeier T, Duda G, Bail HJ (2003) Temporal profile of microvascular disturbances in rat tibial periosteum following closed soft tissue trauma. Langenbecks Arch Surg 388(5):323-330. doi:10.1007/s00423-003-0411-5

11. Varga R, Janovszky A, Szabó A, Garab D, Bodnár D, Boros M, Neunzehn J, Wiesmann HP, Piffkó J (2014) A novel method for in vivo visualization of the microcirculation of the mandibular periosteum in rats. Microcirculation (in press) doi: 10.1111/micc.12128

12. Varga R, Török L, Szabó A, Kovács F, Keresztes M, Varga G, Kaszaki J, Boros M (2008) Effects of colloid solutions on ischemia-reperfusion-induced periosteal microcirculatory and inflammatory reactions: comparison of dextran, gelatin, and hydroxyethyl starch. Crit Care Med 36(10):2828-2837. doi:10. 1097/CCM.0b013e318186ff48

13. Bencsik P, Kupai K, Giricz Z, Görbe A, Pipis J, Murlasits Z, Kocsis GF, Varga-Orvos Z, Puskás LG, Csonka C, Csont T, Ferdinandy P (2010) Role of iNOS and peroxynitrite-matrix metalloproteinase-2 signaling in myocardial late preconditioning in rats. Am J Physiol 299(2):H512-518. doi:10.1152/ajpheart.00052.2010

14. Biasotto M, Chiandussi S, Zacchigna S, Moimas S, Dore F, Pozzato G, Cavalli F, Zanconati F, Contardo L, Giacca M, Di Lenarda R (2010) A novel animal model to study non-spontaneous bisphosphonates osteonecrosis of jaw. J Oral Pathol Med 39(5): 390-396. doi:10.1111/j.1600-0714.2009.00878.x

15. Barba-Recreo P, Del Castillo Pardo de Vera JL, García-Arranz M, Yébenes L, Burgueño M (2013) Zoledronic acid - Related osteonecrosis of the jaws. Experimental model with dental extractions in rats. J Craniomaxillofac Surg pii: S1010-5182(13)00304-1. doi: 10.1016/j.jcms.2013.11.005

16. Marx RE, Cillo JE Jr, Ulloa JJ (2007) Oral bisphosphonate-induced osteonecrosis: risk factors, prediction of risk using serum CTX testing, prevention, and treatment. J Oral Maxillofac Surg 65(12):23972410. doi:10.1016/j.joms.2007.08.003

17. Rodan GA, Fleisch HA (1996) Bisphosphonates: mechanisms of action. J Clin Invest 97(12):2692-2696. doi:10.1172/JCI118722

18. Maruotti N, Corrado A, Neve A, Cantatore FP (2012) Bisphosphonates: effects on osteoblast. Eur J Clin Pharmacol 68(7):1013-1018. doi:10.1007/s00228-012-1216-7

19. Yamashita J, Koi K, Yang DY, McCauley LK (2011) Effect of zoledronate on oral wound healing in rats. Clin Cancer Res 17(6): 1405-1414

20. Kobayashi Y, Hiraga T, Ueda A, Wang L, Matsumoto-Nakano M, Hata K, Yatani H, Yoneda T (2010) Zoledronic acid delays wound healing of the tooth extraction socket, inhibits oral epithelial cell migration, and promotes proliferation and adhesion to hydroxyapatite of oral bacteria, without causing osteonecrosis of the jaw, in mice. J Bone Miner Metab 28(2):165-175. doi:10.1007/s00774-009-0128-9

21. Aguirre JI, Akhter MP, Kimmel DB, Pingel JE, Williams A, Jorgensen M, Kesavalu L, Wronski TJ (2012) Oncologic doses of zoledronic acid induce osteonecrosis of the jaw-like lesions in rice rats (Oryzomys palustris) with periodontitis. J Bone Miner Res 27(10):2130-2143. doi:10.1002/jbmr.1669

22. Wood J, Bonjean K, Ruetz S, Bellahcène A, Devy L, Foidart JM, Castronovo V, Green JR (2002) Novel antiangiogenic effects of the bisphosphonate compound zoledronic acid. J Pharmacol Exp Ther 302(3):1055-1061. doi:10.1124/jpet.102.035295

23. Pabst AM, Ziebart T, Ackermann M, Konerding MA, Walter C (2014) Bisphosphonates' antiangiogenic potency in the development of bisphosphonate-associated osteonecrosis of the jaws: influence on microvessel sprouting in an in vivo 3D Matrigel assay. Clin Oral Investig 18(3):1015-1022. doi:10.1007/s00784-013-1060-x

24. Guevarra CS, Borke JL, Stevens MR, Bisch FC, Zakhary I, Messer R, Gerlach RC, Elsalanty ME (2013) Vascular alterations in the Sprague-Dawley rat mandible during intravenous bisphosphonate therapy. J Oral Implantol (in press) doi: 10.1563/AAID-JOI-D-1300074

25. Reid IR, Bolland MJ, Grey AB (2007) Is bisphosphonate-associated osteonecrosis of the jaw caused by soft tissue toxicity? Bone 41(3): 318-320. doi:10.1016/j.bone.2007.04.196

26. Scheper MA, Badros A, Chaisuparat R, Cullen KJ, Meiller TF (2009) Effect of zoledronic acid on oral fibroblasts and epithelial cells: a potential mechanism of bisphosphonate-associated osteonecrosis. $\mathrm{Br}$ J Haematol 144(5):667-676. doi:10.1111/j.1365-2141.2008.07504.x

27. Açil Y, Möller B, Niehoff P, Rachko K, Gassling V, Wiltfang J, Simon MJ (2012) The cytotoxic effects of three different bisphosphonates in-vitro on human gingival fibroblasts, osteoblasts and osteogenic sarcoma cells. J Craniomaxillofac Surg 40(8):e229 235. doi:10.1016/j.jcms.2011.10.024

28. Yamaguchi K, Motegi K, Iwakura Y, Endo Y (2000) Involvement of interleukin-1 in the inflammatory actions of aminobisphosphonates in mice. Br J Pharmacol 130(7):1646-1654. doi:10.1038/sj.bjp. 0703460

29. Yu YY, Lieu S, Hu D, Miclau T, Colnot C (2012) Site specific effects of zoledronic acid during tibial and mandibular fracture repair. PLoS One 7(2):e31771. doi:10.1158/1078-0432.CCR-10-1614

30. Koch FP, Wunsch A, Merkel C, Ziebart T, Pabst A, Yekta SS, Blessmann M, Smeets R (2011) The influence of bisphosphonates on human osteoblast migration and integrin $\mathrm{aVb} 3 /$ tenascin $\mathrm{C}$ gene expression in vitro. Head Face Med 7(1):4. doi:10.1186/1746-160X7-4

31. Wen D, Qing L, Harrison G, Golub E, Akintoye SO (2011) Anatomic site variability in rat skeletal uptake and desorption of fluorescently labeled bisphosphonate. Oral Dis 17(4):427-432. doi:10.1111/j. 1601-0825.2010.01772.x

32. Marolt D, Cozin M, Vunjak-Novakovic G, Cremers S, Landesberg R (2012) Effects of pamidronate on human alveolar osteoblasts in vitro. J Oral Maxillofac Surg 70(5):1081-1092. doi:10.1016/j.joms.2011. 05.002

33. Çankaya M, Cizmeci Șenel F, Kadioglu Duman M, Muci E, Dayisoylu EH, Balaban F (2013) The effects of chronic zoledronate usage on the jaw and long bones evaluated using RANKL and osteoprotegerin levels in an animal model. Int J Oral Maxillofac Surg 42(9):1134-1139. doi:10.1016/j.ijom.2013.02.008

34. Kuroshima S, Entezami P, McCauley LK, Yamashita J (2014) Early effects of parathyroid hormone on bisphosphonate/steroid-associated compromised osseous wound healing. Osteoporos Int 25(3):11411150. doi:10.1007/s00198-013-2570-8

35. Zhang L, Bail H, Mittlmeier T, Haas NP, Schaser KD (2003) Immediate microcirculatory derangements in skeletal muscle and periosteum after closed tibial fracture. J Trauma 54(5):979-985. doi:10.1097/00005373-200305000-00022

36. Kuiper JW, Forster C, Sun C, Peel S, Glogauer M (2012) Zoledronate and pamidronate depress neutrophil functions and survival in mice. Br J Pharmacol 165(2):532-539. doi:10.1111/j.1476-5381.2011. 01592.x 
37. Yamagishi S, Matsui T, Nakamura K, Takeuchi M (2005) Minodronate, a nitrogen-containing bisphosphonate, inhibits advanced glycation end product-induced vascular cell adhesion molecule-1 expression in endothelial cells by suppressing reactive oxygen species generation. Int J Tissue React 27(4):189-195

38. Favot CL, Forster C, Glogauer M (2013) The effect of bisphosphonate therapy on neutrophil function: a potential biomarker. Int J Oral Maxillofac Surg 42(5):619-626. doi:10.1016/j.ijom.2012.12.011
39. Hagelauer N, Pabst AM, Ziebart T, Ulbrich H, Walter C (2014) In vitro effects of bisphosphonates on chemotaxis, phagocytosis, and oxidative burst of neutrophil granulocytes. Clin Oral Investig (in press) doi: 10.1007/s00784-014-1219-0

40. Eppihimer MJ, Granger DN (1997) Ischemia/reperfusioninduced leukocyte-endothelial interactions in postcapillary venules. Shock 8(1):16-25. doi:10.1097/00024382-19970700000004 